\title{
GENERALIZED SOLUTIONS IN NONLINEAR STOCHASTIC CONTROL PROBLEMS*
}

\author{
F. DUFOUR ${ }^{\dagger}$ AND BORIS M. MILLER ${ }^{\ddagger}$
}

\begin{abstract}
An optimal stochastic control problem is considered for systems with unbounded controls satisfying an integral constraint. It is shown that there exists an optimal control within the class of generalized controls leading to impulse actions. Applying an approach of time transformation, developed recently for deterministic systems, the original control problem is shown to be equivalent to an optimal stopping problem. Moreover, the description of generalized solutions is given in terms of stochastic differential equations governed by a measure.
\end{abstract}

Key words. nonlinear stochastic systems, impulse control, generalized solutions, discontinuous time-change

AMS subject classifications. 49J30, 49N25, 93E20

PII. S0363012900374221

1. Introduction. In this paper, the existence of an optimal control is discussed for the nonlinear stochastic system defined by the following equation:

$$
x_{t} \doteq \zeta+\int_{0}^{t} A\left(s, x_{s}\right) d s+\int_{0}^{t} B\left(s, x_{s}\right) u_{s} d s+\int_{0}^{t} D\left(s, x_{s}\right) d W_{s},
$$

where the functions $A, B$, and $D$ are deterministic, $\left\{W_{t}\right\}$ is a Brownian motion, and $\left\{u_{t}\right\}$ is the control. All the processes are assumed to be defined on a probability space $\left(\Omega, \mathcal{F}, P,\left\{\mathcal{F}_{t}\right\}\right)$. Let $K$ be a closed convex cone. The class of admissible controls, labeled $\mathfrak{C}^{a}$, is defined by the class of $K$-valued, $\left\{\mathcal{F}_{t}\right\}$-predictable processes subject to the following constraint:

$$
\int_{0}^{T}\left|u_{s}\right| d s \leq M
$$

For an admissible control $u$, the cost is given by

$$
J[u]=E\left[g\left(x_{T}\right)\right],
$$

where $g$ is a deterministic function and $T$ is the terminal time.

When the control satisfies condition (2), it is easy to see that the optimal solution may not exist within the class of admissible control (see the example in section 3). Indeed, this constraint (2) implies that the admissible control can be chosen as close as desired to a control of impulsive type. An approach to solve this problem in a deterministic context, based on a time transformation, was originally suggested by Warga [21] and has been actively developed recently (see, for example, the survey [15]).

\footnotetext{
* Received by the editors June 19, 2000; accepted for publication (in revised form) November 1, 2001; published electronically February 14, 2002. This research was supported by a CNRS/Russian Academy of Sciences cooperation (PECO/NEI 9570) and in part by the Nonlinear Control Network and by Russian Basic Investigation Foundation grant 99-01-01-088.

http://www.siam.org/journals/sicon/40-6/37422.html

${ }^{\dagger}$ LaBRI, Universite Bordeaux I, 351 cours de la Liberation, 33405 Talence Cedex, France (dufour@ labri.u-bordeaux.fr).

${ }^{\ddagger}$ Institute for Information Transmission Problems, 19 Bolshoy Karetny per., Moscow 101447, Russia (bmiller@iitp.ru).
} 
In the stochastic context, this approach was introduced by Miller and Runggaldier in [17] to solve a special case of the problem studied in the present work. In this context, it appears necessary to introduce a new concept to describe the limit of a sequence of control processes subject to the constraint (2); this is the so-called generalized control. (For a more precise exposition, see Definition 3.1.) Similarly, the limit of a sequence of solutions of (1) is defined as a generalized solution. These definitions of generalized control and generalized solution are taken from the deterministic context (see, for example, $[1,16,18])$.

Our aim is to characterize the value of $\inf _{u \in \mathfrak{C}^{a}} J[u]$. By introducing the class of admissible generalized controls, labeled $\overline{\mathfrak{C}}^{a}$, it is shown that $\inf _{u \in \mathfrak{C}^{a}} J[u]=\inf _{u \in \overline{\mathfrak{C}}^{a}} J[u]$. The characterization of $\inf _{u \in \mathfrak{C}^{a}} J[u]$ will be completed when it is shown that the there exists an optimal generalized control $u^{*} \in \overline{\mathfrak{C}}^{a}$ such that inf ${ }_{u \in \overline{\mathfrak{C}}^{a}} J[u]=J\left[u^{*}\right]$. It proves that there exists an optimal generalized control for the original control problem, justifying, therefore, the introduction of this class of process, $\overline{\mathfrak{C}}^{a}$. This existence result is obtained by using a time transformation to convert the original control problem into an optimal stopping problem. Moreover, the representation of the generalized solution is given in terms of a stochastic differential equation governed by a measure. This important property enhances the link existing between this control problem and the class of singular control problems.

Singular stochastic control problems have recently received considerable attention in the literature (see $[9,10,22]$ and the references therein). However, until now the theoretical basis for this kind of stochastic control problem was restricted to the class of systems where the gain of the singular control does not depend on the state process (see, for example, $[9,10]$ and the references therein). Therefore, our work can be considered as a first attempt to extend these results in the case where the gain of the singular control may depend on the state process. Other extensions of our approach are already planned, and in [4] it will be shown how this method can be applied to re-examine the singular problem studied in [9]. It must be pointed out that the control problem defined in (1)-(3) cannot be solved directly by using the results in [8, Theorem 4.7]. Our work can be generalized in several directions by adding soft constraints and considering the optimal stopping problem.

The paper is organized as follows. In section 2, we formulate the original control problem. The concept of generalized control is introduced in section 3 by analogy with the deterministic case. It is shown that the infimum of the expected cost over the class of admissible controls and the infimum over the class of admissible generalized controls are the same (see Proposition 3.2). Section 4 contains the description of the time transformation and introduces an auxiliary control problem that will be shown to be equivalent to the original one. On the basis of known results [8], the existence theorem is proved for the auxiliary problem. A consequence of this result is derived in section 5 and shows that there exists an optimal generalized control for the original control problem. Its representation is given in terms of a stochastic differential equation governed by a measure. In the appendix, some technical results are derived.

We introduce the following notation and terminology.

Notation. $\mathbb{N}_{N}$ is the set of the first $N$ integers, that is, $\mathbb{N}_{N}=\{1, \ldots, i, \ldots, N\}$. $\mathbb{R}_{+} \doteq\{x \in \mathbb{R}: x \geq 0\}$. The $i$ th component of a vector $M$ is denoted by $M^{i}$. The symbol |.| is used to denote the norm of vectors and matrices. If $X$ is a normed space, then for $R>0$ the set $B_{R}(X)$ is defined by $\{x \in X:|x|<R\}$ and $\bar{B}_{R}(X) \doteq\{x \in$ $X:|x| \leq R\}$. (') denotes the transpose operation. $0_{n} \in \mathbb{R}^{n}$ is the zero vector. The indicator function of a set $A$ is defined as $I_{A}(x)$. On a probability space $(\Omega, \mathcal{F}, P)$, 
the mathematical expectation will be denoted by $E_{P}[$.$] .$

In order to define the state processes, let us introduce the following data:

- $K$ is a subset of $\mathbb{R}^{p}$.

- $A: \mathbb{R}_{+} \times \mathbb{R}^{n} \rightarrow \mathbb{R}^{n}$

- $B: \mathbb{R}_{+} \times \mathbb{R}^{n} \rightarrow \mathbb{R}^{n \times p}$.

- $D: \mathbb{R}_{+} \times \mathbb{R}^{n} \rightarrow \mathbb{R}^{n \times m}$.

- $g: \mathbb{R}^{n} \rightarrow \mathbb{R}_{+}$.

- $\zeta$ is a fixed vector in $\mathbb{R}^{n}$.

- $T$ and $M$ are fixed real numbers.

- $G: \mathbb{R}_{+} \rightarrow \overline{\mathbb{R}}_{+}$such that $G(T)=0$ and $G(t)=\infty$ for $t \neq T$.

The following assumptions will be used in the paper.

(A.1) There are constants $L_{1}$ and $L_{2}$ such that for all $t, s \in \mathbb{R}_{+}$and $x, y \in \mathbb{R}^{n}$

$$
\begin{gathered}
|A(t, x)|+|B(t, x)|+|D(t, x)| \leq L_{1}(1+|x|), \\
|A(t, x)-A(s, y)|+|B(t, x)-B(s, y)|+|D(t, x)-D(s, y)| \leq L_{2}(|x-y|+|t-s|) .
\end{gathered}
$$

(A.2) The function $g$ is continuous, and there exist a constant $L_{3}$ and a positive integer $q$ such that

$$
|g(x)|^{2} \leq L_{3}\left(1+|x|^{q}\right)
$$

(A.3) $K$ is a closed cone which is convex.

(A.4) For all $(t, x) \in[0, T) \times \mathbb{R}^{n}$, the set $K(t, x)$ defined by

$$
K(t, x) \doteq\left\{\left((1-|\theta|) A(t, x)+B(t, x) \theta,(1-|\theta|) D(t, x) D(t, x)^{\prime},|\theta|\right): \theta \in \bar{B}_{1}(K)\right\}
$$

is convex.

2. Problem statement. In this section, we formulate the stochastic control problem presented in the introduction using the formulation described in Haussmann and Lepeltier [8] and El Karoui, Nguyen, and Jeanblanc-Picqué [5].

Definition 2.1. A control is defined by the term

$$
C \doteq\left(\Omega, \mathcal{F}, P,\left\{\mathcal{F}_{t}\right\},\left\{u_{t}\right\},\left\{W_{t}\right\},\left\{x_{t}\right\}\right)
$$

where the following hold:

(i) $(\Omega, \mathcal{F}, P)$ is a complete probability space with a right continuous complete filtration $\left\{\mathcal{F}_{t}\right\}$.

(ii) $\left\{u_{t}\right\}$ is a $K$-valued, $\left\{\mathcal{F}_{t}\right\}$-predictable process such that

$$
\int_{0}^{T}\left|u_{s}\right| d s \leq M
$$

(iii) $\left\{W_{t}\right\}$ is an $\left\{\mathcal{F}_{t}\right\}$ standard m-dimensional Brownian motion.

(iv) $\left\{x_{t}\right\}$ is an $\mathbb{R}^{n}$-valued, $\left\{\mathcal{F}_{t}\right\}$ progressively measurable process such that

$$
\begin{aligned}
(\forall t \in[0, T]), x_{t} \doteq & +\int_{0}^{t} A\left(s, x_{s}\right) d s+\int_{0}^{t} B\left(s, x_{s}\right) u_{s} d s \\
& +\int_{0}^{t} D\left(s, x_{s}\right) d W_{s} .
\end{aligned}
$$


We write $\mathfrak{C}$ for the set of controls satisfying the previous conditions.

The cost is given by

$$
J[C] \doteq E_{P}\left[g\left(x_{T}\right)\right]
$$

The set $\mathfrak{C}^{a}$ of admissible controls is defined by

$$
\mathfrak{C}^{a} \doteq\{C \in \mathfrak{C}: J[C]<\infty\}
$$

We shall consider as a control objective the minimization of $J[C]$ on $\mathfrak{C}^{a}$.

As already pointed out in the introduction, since we do not assume any conditions such as the coercivity condition (see (3.5) in [8]), the existence of an optimal control for the previous problem cannot be claimed using the approach described in [8]. Before presenting the concept of generalized control, let us derive the following technical lemma.

LEMma 2.2. The stochastic differential equation (5), where $\left\{u_{t}\right\}$ satisfies item (ii) of Definition 2.1, has a unique solution such that

$$
(\forall q \in \mathbb{N}) \quad E_{P}\left[\sup _{t \in[0, T]}\left|x_{t}\right|^{2 q}\right]<D,
$$

where $D$ is a constant.

Proof. Using (A.1) and Theorem 7, page 197 in [19], the existence and the uniqueness of the solution are straightforward. The proof of (8) is given in the appendix. We cannot use standard arguments to derive it since the process $\left\{u_{t}\right\}$ may not be bounded but satisfies the inequality (4).

3. Generalized controls. An optimal control may not exist within the class of ordinary admissible controls $\mathfrak{C}^{a}$. An example is now presented in order to illustrate this assertion. A deterministic problem is considered where $T=1, M=1$, the control $u_{t} \in K \doteq \mathbb{R}_{+}$, and the state satisfies the following equation:

$$
(\forall t \in[0,1]) \quad x_{t} \doteq \int_{0}^{t}\left(u_{s}-x_{s}\right) d s
$$

The aim is to minimize the cost $J[C]=\left(1-x_{1}\right)^{2}$. It is easy to show that $x_{1}=$ $\int_{0}^{1} e^{(s-1)} u_{s} d s$, and, by using the fact that $\int_{0}^{1} u_{s} d s \leq 1$, it follows that for any admissible control $J[C]>0$.

Now let us introduce the sequence of admissible controls

$$
u_{t}^{n}= \begin{cases}0 & \text { for } 0 \leq t \leq 1-\frac{1}{n} \\ n & \text { for } 1-\frac{1}{n}<t \leq 1\end{cases}
$$

Clearly, $\int_{0}^{1} u_{s}^{n} d s=1$, and the cost $J\left[C^{n}\right]$ associated to $u^{n}$ is equal to $\left[1-n\left(1-e^{-\frac{1}{n}}\right)\right]^{2}$. Consequently, $\lim _{n \rightarrow \infty} J\left[C^{n}\right]=0$, showing that an optimal control does not exist within the class of ordinary admissible controls $\mathfrak{C}^{a}$. This is a consequence of the discontinuous behavior of the minimizing sequence $\left\{u_{t}^{n}\right\}$ at $t=1$.

In order to characterize $\inf _{C \in \mathfrak{C}^{a}} J[C]$, we introduce the concept of generalized control, labeled $C^{g}$, and its associated class of admissible controls $\overline{\mathfrak{C}^{a}}$. Moreover, the correspondence between $\inf _{C \in \mathfrak{C}^{a}} J[C]$ and $\inf _{C^{g} \in \overline{\mathfrak{C}}^{a}} J\left[C^{g}\right]$ is given in Proposition 3.2, justifying the introduction of this new class of controls $\overline{\mathfrak{C}}^{a}$. 
Definition 3.1. A generalized control is defined by the term

$$
C^{g} \doteq\left(\Omega, \mathcal{F}, P,\left\{\mathcal{F}_{t}\right\},\left\{U_{t}\right\},\left\{W_{t}\right\},\left\{X_{t}\right\}\right)
$$

where the following hold:

(i) $(\Omega, \mathcal{F}, P)$ is a complete probability space with a right continuous complete filtration $\left\{\mathcal{F}_{t}\right\}$.

(ii) $\left\{U_{t}\right\}$ is a $K$-valued, corlol, $\left\{\mathcal{F}_{t}\right\}$ progressively measurable process satisfying

$$
\operatorname{Var}_{[0, T]}\left[U_{t}\right] \leq M, \quad U_{t}-U_{s} \in K \quad \text { for } t \geq s
$$

(iii) $\left\{W_{t}\right\}$ is an $\left\{\mathcal{F}_{t}\right\}$ standard $m$-dimensional Brownian motion.

(iv) $\left\{X_{t}\right\}$ is an $\mathbb{R}^{n}$-valued, corlol, $\left\{\mathcal{F}_{t}\right\}$ progressively measurable semimartingale such that the continuous part of $\left\{X_{t}\right\}$ satisfies

$$
\begin{aligned}
(\forall t \in[0, T]) \quad X_{t}^{c} \doteq \zeta & +\int_{0}^{t} A\left(s, X_{s}\right) d s+\int_{0}^{t} B\left(s, X_{s}\right) d U_{s}^{c} \\
& +\int_{0}^{t} D\left(s, X_{s}\right) d W_{s} .
\end{aligned}
$$

(v) There exists a sequence $\left\{C^{n}\right\}_{n \in \mathbb{N}}$ defined by

$$
C^{n} \doteq\left(\Omega, \mathcal{F}, P,\left\{\mathcal{F}_{t}^{n}\right\},\left\{u_{t}^{n}\right\},\left\{W_{t}^{n}\right\},\left\{x_{t}^{n}\right\}\right)
$$

such that

$$
(\forall n \in \mathbb{N}) \quad C^{n} \in \mathfrak{C}^{a}
$$

and

$$
\begin{aligned}
(\forall t \in[0, T)) \quad X_{t} & =\limsup _{\substack{s \rightarrow t \\
s>t}} \lim _{n \rightarrow \infty} x_{s}^{n}, \quad P-\text { a.s. }, \\
\text { and } \quad X_{T} & =\lim _{n \rightarrow \infty} x_{T}^{n}, \quad P-\text { a.s. }
\end{aligned}
$$

We write $\overline{\mathfrak{C}}$ for the set of controls satisfying the previous conditions.

The cost is given by

$$
J\left[C^{g}\right] \doteq E_{P}\left[g\left(X_{T}\right)\right]
$$

The set $\overline{\mathfrak{C}}^{a}$ of admissible controls is defined by

$$
\overline{\mathfrak{C}}^{a} \doteq\left\{C^{g} \in \overline{\mathfrak{C}}: J\left[C^{g}\right]<\infty\right\}
$$

Note that the discontinuous part of $\left\{X_{t}\right\}$ is generated by the discontinuous part of $\left\{U_{t}\right\}$.

The following result provides a correspondence between the sets of control $\mathfrak{C}^{a}$ and $\overline{\mathfrak{C}}^{a}$. Its proof is an immediate consequence of the definitions of $\mathfrak{C}^{a}$ and $\overline{\mathfrak{C}}^{a}$ and assumption (A.2).

Proposition 3.2. The set of control $\mathfrak{C}^{a}$ is a subset of $\overline{\mathfrak{C}}^{a}$, and

$$
\inf _{C \in \mathfrak{C}^{a}} J[C]=\inf _{C^{g} \in \overline{\mathfrak{C}}^{a}} J\left[C^{g}\right] .
$$


4. Time transformation and the auxiliary control problem. In this section, we introduce an auxiliary control problem which is given in terms of an optimal stopping problem (see Definition 4.1). It is shown in Corollary 4.16 that this problem is equivalent to the initial one. A key property of the auxiliary control problem is that the controls take their values in a compact set.

DeFinition 4.1. An auxiliary control is defined by the term

$$
\Psi \doteq\left(\Omega, \mathcal{F}, P,\left\{\mathcal{G}_{t}\right\},\left\{\theta_{t}\right\},\left\{V_{t}\right\},\left\{\Lambda_{t}\right\}, \gamma\right),
$$

where the following hold:

(i) $(\Omega, \mathcal{F}, P)$ is a complete probability space with a right continuous complete filtration $\left\{\mathcal{G}_{t}\right\}$

(ii) $\left\{\theta_{t}\right\}$ is a $\bar{B}_{1}(K)$-valued, $\left\{\mathcal{G}_{t}\right\}$-predictable process.

(iii) $\left\{V_{t}\right\}$ is a $\left\{\mathcal{G}_{t}\right\}$ standard $m$-dimensional Brownian motion.

(iv) $\gamma$ is a $\left\{\mathcal{G}_{t}\right\}$ stopping time such that

$$
\gamma \leq T+M
$$

(v) $\left\{\Lambda_{t} \doteq\left(\eta_{t}, \xi_{t}^{\prime}\right)^{\prime}\right\}$ is an $\mathbb{R}^{n+1}$-valued, $\left\{\mathcal{G}_{t}\right\}$ progressively measurable process such that

$$
\begin{aligned}
\eta_{t} \doteq t & -\int_{0}^{t}\left|\theta_{s}\right| d s \\
\xi_{t} \doteq \zeta & +\int_{0}^{t}\left(1-\left|\theta_{s}\right|\right) A\left(\eta_{s}, \xi_{s}\right) d s+\int_{0}^{t} B\left(\eta_{s}, \xi_{s}\right) \theta_{s} d s \\
& +\int_{0}^{t} \sqrt{1-\left|\theta_{s}\right|} D\left(\eta_{s}, \xi_{s}\right) d V_{s}
\end{aligned}
$$

for $t \in[0, \gamma]$.

We write $\bar{\Upsilon}$ for the set of controls satisfying the previous conditions.

The cost is given by

$$
\mathcal{M}[\Psi] \doteq E_{P}\left[g\left(\xi_{\gamma}\right)+G\left(\eta_{\gamma}\right)\right]
$$

The set $\bar{\Upsilon}^{a}$ of admissible auxiliary controls is defined by

$$
\bar{\Upsilon}^{a} \doteq\{\Psi \in \bar{\Upsilon}: \mathcal{M}[\Psi]<\infty\} .
$$

Our aim is to show the equivalence between the auxiliary and the initial control problems. However, we first show the existence of an optimal control for the auxiliary problem.

THEOREM 4.2. For the auxiliary control problem there exists an optimal control $\Theta^{*}$ :

$$
\inf _{\Psi \in \bar{\Upsilon}^{a}} \mathcal{M}[\Psi]=\mathcal{M}\left[\Theta^{*}\right] \quad \text { and } \quad \Theta^{*} \in \bar{\Upsilon}^{a}
$$

Proof. Applying Corollary 4.8 in [8], it follows that there exist a probability space $(\Omega, \widetilde{\mathcal{F}}, \widetilde{P})$ and a filtration $\left\{\widetilde{\mathcal{G}}_{t}\right\}$ such that

- $\left\{\widetilde{\theta}_{t}\right\}$ is a $\bar{B}_{1}(K)$-valued, $\left\{\widetilde{\mathcal{G}}_{t}\right\}$ progressively measurable process,

- $\left\{V_{t}\right\}$ is a $\left\{\widetilde{\mathcal{G}}_{t}\right\}$ standard $m$-dimensional Brownian motion, 
and

$$
E_{\widetilde{P}}\left[g\left(\xi_{\gamma}\right)\right] \leq \inf _{\Psi \in \bar{\Upsilon}^{a}} \mathcal{M}[\Psi],
$$

where $\gamma$ is a $\left\{\widetilde{\mathcal{G}}_{t}\right\}$ stopping time and

$$
\begin{aligned}
& \eta_{t}=t-\int_{0}^{t}\left|\widetilde{\theta}_{s}\right| d s \\
& \xi_{t}=\zeta+\int_{0}^{t}\left(1-\left|\widetilde{\theta}_{s}\right|\right) A\left(\eta_{s}, \xi_{s}\right) d s+\int_{0}^{t} B\left(\eta_{s}, \xi_{s}\right) \widetilde{\theta}_{s} d s+\int_{0}^{t} \sqrt{1-\left|\widetilde{\theta}_{s}\right|} D\left(\eta_{s}, \xi_{s}\right) d V_{s} .
\end{aligned}
$$

In (21), we do not have an equality because in the control problem studied by Haussmann and Lepeltier [8] the set of admissible controls is defined on the set of progressively measurable processes and for an arbitrary probability space. In our case, the admissible controls are defined in the smaller set of predictable processes and on a probability space that must satisfy the usual hypotheses (completion and right continuity). However, using Lemmas A.1 and A.2, it can be shown that there exists a new probability space $\left(\Omega, \mathcal{F}, P,\left\{\mathcal{G}_{t}\right\}\right)$ satisfying the usual hypotheses based on a modification of $\left(\Omega, \widetilde{\mathcal{F}}, \widetilde{P},\left\{\widetilde{\mathcal{G}}_{t}\right\}\right)$. Moreover, the existence of a $\bar{B}_{1}(K)$-valued, $\left\{\mathcal{G}_{t}\right\}$-predictable process $\left\{\theta_{t}\right\}$ such that

$$
\Theta^{*} \doteq\left(\Omega, \mathcal{F}, P,\left\{\mathcal{G}_{t}\right\},\left\{\theta_{t}\right\},\left\{V_{t}\right\},\left\{\left(\eta_{t}, \xi_{t}^{\prime}\right)^{\prime}\right\}, \gamma\right) \in \bar{\Upsilon}^{a} \quad \text { and } \quad \mathcal{M}\left[\Theta^{*}\right] \leq \inf _{\Psi \in \bar{\Upsilon}^{a}} \mathcal{M}[\Psi]
$$

is guaranteed by Lemma A.3.

Consequently, we have $\mathcal{M}\left[\Theta^{*}\right]=\inf _{\Psi \in \bar{\Upsilon}^{a}} \mathcal{M}[\Psi]$, which gives the result.

In order to establish the correspondence between the auxiliary control problem and the initial one, we need to introduce the following subset of $\bar{\Upsilon}^{a}$, labeled $\Upsilon^{a}$ (see Definition 4.3). We prove in Theorem 4.9 that

$$
\begin{aligned}
\inf _{\Psi \in \Upsilon^{a}} \mathcal{M}[\Psi] & =\min _{\Psi \in \bar{\Upsilon}^{a}} \mathcal{M}[\Psi] \\
& =\mathcal{M}\left[\Theta^{*}\right] .
\end{aligned}
$$

Then it is shown in Theorem 4.15 that

$$
\inf _{C \in \mathfrak{C}^{a}} J[C]=\inf _{\Psi \in \Upsilon^{a}} \mathcal{M}[\Psi] .
$$

Therefore, combining (22) and (23), the main result of this section (see Corollary 4.16) will follow; that is,

$$
\inf _{C \in \mathfrak{C}^{a}} J[C]=\mathcal{M}\left[\Theta^{*}\right] .
$$

The rest of this section is devoted to the proofs of relations (22) and (23).

First, in order to show that (22) holds, we prove that for any control $\Psi \in \bar{\Upsilon}^{a}$ there exists a sequence of controls $\left\{\Psi^{n}\right\}$ in $\Upsilon^{a}$ such that $\lim _{n \rightarrow \infty} \mathcal{M}\left[\Psi^{n}\right]=\mathcal{M}[\Psi]$. This is not a trivial consequence of the closure of $\Upsilon^{a}$ by $\bar{\Upsilon}^{a}$ since it is necessary to approximate the stopping time $\gamma$. Now we need the following definitions and technical results.

Definition 4.3. Let us introduce the set $\Upsilon \subset \bar{\Upsilon}$ :

$$
\Psi \doteq\left(\Omega, \mathcal{F}, P,\left\{\mathcal{G}_{t}\right\},\left\{\theta_{t}\right\},\left\{V_{t}\right\},\left\{\Lambda_{t}\right\}, \gamma\right) \in \Upsilon \Longleftrightarrow\left\{\begin{array}{l}
\Psi \in \bar{\Upsilon} \\
\text { and } \\
\left\{\theta_{t}\right\} \text { is a } B_{1}(K) \text {-valued process }
\end{array}\right.
$$


and the corresponding set of admissible controls

$$
\Upsilon^{a}=\Upsilon \cap \bar{\Upsilon}^{a}
$$

Definition 4.4. For $\Psi \doteq\left(\Omega, \mathcal{F}, P,\left\{\mathcal{G}_{t}\right\},\left\{\theta_{t}\right\},\left\{V_{t}\right\},\left\{\Lambda_{t}=\left(\eta_{t}, \xi_{t}^{\prime}\right)^{\prime}\right\}, \gamma\right) \in \bar{\Upsilon}^{a}$, define on $(\Omega, \mathcal{F}, P)$

$$
\begin{aligned}
\nu^{n} & \doteq \inf \left\{t \geq 0: t-\int_{0}^{t} \frac{n}{n+1}\left|\theta_{s}\right| d s \geq \frac{n T}{n+1}\right\}, \\
\nu & \doteq \inf \left\{t \geq 0: \eta_{t} \geq T\right\} \\
\alpha^{n} & \doteq \frac{T+M-\nu^{n}-\frac{T}{n+1}}{T+M-\nu^{n}} .
\end{aligned}
$$

Lemma 4.5. If $\Psi \doteq\left(\Omega, \mathcal{F}, P,\left\{\mathcal{G}_{t}\right\},\left\{\theta_{t}\right\},\left\{V_{t}\right\},\left\{\Lambda_{t}=\left(\eta_{t}, \xi_{t}^{\prime}\right)^{\prime}\right\}, \gamma\right)$ is an element of $\bar{\Upsilon}^{a}$, then $\nu$ and $\nu^{n}$ are $\left\{\mathcal{G}_{t}\right\}$ stopping times (for all $n \in \mathbb{N}$ ) and

$$
\lim _{n \rightarrow \infty} \nu^{n}=\nu, \quad P-\text { a.s. }
$$

and

$$
0 \leq \alpha^{n}<1
$$

Proof. See the appendix.

Using Lemma 4.5, we can now show that a sequence of control $\left\{\Psi^{n}\right\}$ in $\Upsilon^{a}$ can be constructed from any element $\Psi$ in $\bar{\Upsilon}^{a}$ as described below.

Proposition 4.6. Assume that $\Psi \doteq\left(\Omega, \mathcal{F}, P,\left\{\mathcal{G}_{t}\right\},\left\{\theta_{t}\right\},\left\{V_{t}\right\},\left\{\Lambda_{t}=\left(\eta_{t}, \xi_{t}^{\prime}\right)^{\prime}\right\}, \gamma\right)$ is an element of $\bar{\Upsilon}^{a}$. Define the sequence $\left\{\Psi^{n}\right\}_{n \in \mathbb{N}}$ by

$$
\Psi^{n} \doteq\left(\Omega, \mathcal{F}, P,\left\{\mathcal{G}_{t}\right\},\left\{\theta_{t}^{n}\right\},\left\{V_{t}\right\},\left\{\Lambda_{t}^{n}=\left(\eta_{t}^{n}, \xi_{t}^{n \prime}\right)^{\prime}\right\}, \gamma^{n}\right),
$$

where

(31) $\theta_{t}^{n} \doteq \frac{n}{n+1} \theta_{t} I_{\llbracket 0, \nu^{n} \rrbracket}+\alpha^{n} e_{1} I_{\left.\rrbracket \nu^{n}, \nu\right]}+\alpha^{n} \theta_{t} I_{\rrbracket \nu, \gamma \rrbracket} \quad\left(e_{1} \doteq(1,0, \ldots, 0)^{\prime} \in \mathbb{R}^{p}\right)$,

(32) $\eta_{t}^{n} \doteq t-\int_{0}^{t}\left|\theta_{s}^{n}\right| d s$,

$$
\xi_{t}^{n} \doteq \zeta+\int_{0}^{t}\left(1-\left|\theta_{s}^{n}\right|\right) A\left(\eta_{s}^{n}, \xi_{s}^{n}\right) I_{\left\{s \leq \gamma^{n}\right\}} d s+\int_{0}^{t} B\left(\eta_{s}^{n}, \xi_{s}^{n}\right) \theta_{s}^{n} I_{\left\{s \leq \gamma^{n}\right\}} d s
$$

$$
+\int_{0}^{t} \sqrt{1-\mid \theta_{s}^{n \mid}} D\left(\eta_{s}^{n}, \xi_{s}^{n}\right) I_{\left\{s \leq \gamma^{n}\right\}} d V_{s}
$$

(34) $\gamma^{n} \doteq \inf \left\{t \geq 0: \eta_{t}^{n}>T\right\}$.

Then $\Psi^{n} \in \Upsilon^{a}$ for all $n \in \mathbb{N}$.

Proof. From Lemma 4.5 and assumption (A.2), it follows that for all $n \in \mathbb{N}$, $\left\{\theta_{t}^{n}\right\}$ is a $B_{1}(K)$-valued process. Moreover, using the fact that $\alpha^{n}$ is measurable with respect to $\mathcal{G}_{\nu^{n}}, \mathcal{G}_{\nu^{n}} \subset \mathcal{G}_{\nu}$, and Corollary 6.34 in [6], it follows easily that for all $n \in \mathbb{N}$, the process $\left\{\theta_{t}^{n}\right\}$ is $\left\{\mathcal{G}_{t}\right\}$-predictable. From the definitions of $\left\{\eta_{t}^{n}\right\}$ and $\gamma^{n}$, we obtain that $\eta_{T+M}^{n} \geq \eta_{\gamma^{n}}^{n}$. Therefore, we have that

$$
\gamma^{n} \leq T+M
$$


because $\left\{\eta_{t}^{n}\right\}$ is a strictly increasing process.

Now, applying Theorem 7, page 197 in [19], it is easy to see that (33) has a unique solution. Therefore, for all $n \in \mathbb{N}$ the control $\Psi^{n}$ satisfies all of the conditions of Definition 4.3.

Clearly, we have $E_{P}\left[G\left(\eta_{\gamma^{n}}^{n}\right)\right]=0$ and

$$
E_{P}\left[\sup _{s \leq T+M}\left|\xi_{s}^{n}\right|^{p}\right] \leq C
$$

for a constant $C$ depending on $p$ but independent of $n$.

Combining hypothesis (A.2), the previous inequalities, and (35), we obtain that

$$
\mathcal{M}\left[\Psi^{n}\right]=E_{P}\left[g\left(\xi_{\gamma^{n}}^{n}\right)\right]<\infty,
$$

and so $\Psi^{n} \in \Upsilon^{a}$ for all $n \in \mathbb{N}$.

In order to derive the convergence of $\mathcal{M}\left[\Psi^{n}\right]$ to the cost function $\mathcal{M}[\Psi]$, we need the following technical lemma.

LEMma 4.7. Assume that $\Psi \doteq\left(\Omega, \mathcal{F}, P,\left\{\mathcal{G}_{t}\right\},\left\{\theta_{t}\right\},\left\{V_{t}\right\},\left\{\Lambda_{t}=\left(\eta_{t}, \xi_{t}^{\prime}\right)^{\prime}\right\}, \gamma\right)$ is an element of $\bar{\Upsilon}^{a}$. Then

$$
0 \leq \gamma^{n}-\gamma \leq \frac{T}{n+1}
$$

and

$$
E_{P}\left[\left|\int_{0}^{\gamma}\right| \theta_{s}-\left.\left.\theta_{s}^{n}\right|^{2} d s\right|^{2}+\int_{0}^{\gamma}\left|\eta_{s}-\eta_{s}^{n}\right|^{2} d s\right] \leq C\left\{\frac{1}{(n+1)^{2}}+E_{P}\left[\left|\nu-\nu^{n}\right|^{2}\right]\right\}
$$

for a constant $C$ independent of $n$.

Proof. See the appendix.

Finally, based on the previous lemma, we can prove that the sequence $\left\{\Psi^{n}\right\}$ satisfies the desired property.

Proposition 4.8. Assume that $\Psi \doteq\left(\Omega, \mathcal{F}, P,\left\{\mathcal{G}_{t}\right\},\left\{\theta_{t}\right\},\left\{V_{t}\right\},\left\{\Lambda_{t}=\left(\eta_{t}, \xi_{t}^{\prime}\right)^{\prime}\right\}, \gamma\right)$ is an element of $\bar{\Upsilon}^{a}$. Then the sequence $\left\{\Psi^{n}\right\}$ in $\Upsilon^{a}$ satisfies

$$
\lim _{n \rightarrow \infty} \mathcal{M}\left[\Psi^{n}\right]=\mathcal{M}[\Psi]
$$

Proof. Let us introduce the following equation:

$$
\begin{aligned}
\chi_{t}=\zeta & +\int_{0}^{t}\left(1-\left|\theta_{s}\right|\right) A\left(\eta_{s}, \chi_{s}\right) I_{\{s \leq \gamma\}} d s+\int_{0}^{t} B\left(\eta_{s}, \chi_{s}\right) \theta_{s} I_{\{s \leq \gamma\}} d s \\
& +\int_{0}^{t} \sqrt{1-\left|\theta_{s}\right|} D\left(\eta_{s}, \chi_{s}\right) I_{\{s \leq \gamma\}} d V_{s} .
\end{aligned}
$$

Applying Theorem 7, page 197 in [19], it is easy to see that this equation has a unique solution.

By using Doob's inequality and Gronwall's lemma, it is easy to show that there exists a constant $\bar{C}$ such that
(42) $(\forall t \in[0, T+M])$

$$
E_{P}\left[\sup _{s \leq t}\left|\chi_{s}-\xi_{s}^{n}\right|^{2}\right] \leq \bar{C}\left[\frac{1}{n+1}+\sqrt{E_{P}\left[\left|\nu^{n}-\nu\right|^{2}\right]}\right] .
$$


Moreover, we clearly have $\chi_{T+M}=\xi_{\gamma}$ and $\xi_{T+M}^{n}=\xi_{\gamma^{n}}^{n}$, and so

$$
E_{P}\left[\left|\xi_{\gamma}-\xi_{\gamma^{n}}^{n}\right|^{2}\right] \leq \bar{C}\left[\frac{1}{n+1}+\sqrt{E_{P}\left[\left|\nu^{n}-\nu\right|^{2}\right]}\right]
$$

The sequence $\nu^{n}$ is bounded by $T+M$, and so it is uniformly integrable. Therefore, using Lemma 4.5, we have that $\lim _{n \rightarrow \infty} E_{P}\left[\left|\nu^{n}-\nu\right|^{2}\right]$. With (43), we obtain that $g\left(\xi_{\gamma^{n}}^{n}\right) \longrightarrow_{n \rightarrow \infty}^{P} g\left(\xi_{\gamma}\right)$ since the function $g$ is continuous. Clearly, the sequence $\left\{g\left(\xi_{\gamma^{n}}^{n}\right)\right\}$ is uniformly integrable, and so

$$
\lim _{n \rightarrow \infty} E_{P}\left[g\left(\xi_{\gamma^{n}}^{n}\right)\right]=E_{P}\left[g\left(\xi_{\gamma}\right)\right]
$$

giving the result.

In conclusion, we obtain the following result.

THEOREM 4.9. Let $\Theta^{*} \in \bar{\Upsilon}^{a}$ be the optimal control for the auxiliary control problem. Then

$$
\inf _{\Psi \in \Upsilon^{a}} \mathcal{M}[\Psi]=\mathcal{M}\left[\Theta^{*}\right]
$$

Proof. The existence of $\Theta^{*}$ has been shown in Theorem 4.2. Since $\Upsilon^{a} \subset \bar{\Upsilon}^{a}$, we clearly have

$$
\inf _{\Psi \in \Upsilon^{a}} \mathcal{M}[\Psi] \geq \mathcal{M}\left[\Theta^{*}\right]
$$

However, using Proposition 4.8, the result follows.

Now let us show that (23) holds. Its proof is given in Theorem 4.15 and is based on Propositions 4.12 and 4.14. Here we use two time transformations which establish the correspondence between $\mathfrak{C}^{a}$ and $\Upsilon^{a}$.

Let us introduce the following time-change.

Lemma 4.10. Let $\left(\Omega, \mathcal{F}, P,\left\{\mathcal{F}_{t}\right\},\left\{u_{t}\right\},\left\{W_{t}\right\},\left\{z_{t}=\left(y_{t}, x_{t}^{\prime}\right)^{\prime}\right\}\right)$ be an element of $\mathfrak{C}$, and let $\left\{\Gamma_{t}\right\}$ be the process defined by

$$
\Gamma_{t} \doteq t+\int_{0}^{t}\left|u_{s \wedge T}\right| d s .
$$

Denote by $\left\{\Phi_{t}\right\}$ the right inverse of $\Gamma$ :

$$
\Phi_{t} \doteq \inf \left\{s \geq 0: \Gamma_{s}>t\right\} \text {. }
$$

Then $\left\{\Phi_{t}\right\}$ is a continuous time-change satisfying the following properties:

(i) $\left(\forall t \in \mathbb{R}_{+}\right) \quad \Phi_{\Gamma_{t}}=t \quad$ and $\quad \Gamma_{\Phi_{t}}=t$.

(ii) $\left(\forall t \in \mathbb{R}_{+}\right) \quad \Phi_{t}=\int_{0}^{t} \frac{1}{1+\left|u_{\Phi_{s} \wedge T}\right|} d s$.

Proof. Item (i) is obvious. Differentiating the second equality in (i) and using (46), item (ii) follows easily.

REMARK 4.11. An immediate consequence of the previous lemma is the following assertion:

$$
\left(\forall t \in\left[0, \Gamma_{T}\right]\right) \quad \Phi_{t}=\int_{0}^{t} \frac{1}{1+\left|u_{\Phi_{s}}\right|} d s
$$

which will be used repeatedly in what follows. 
The following proposition shows that for any control $C \in \mathfrak{C}^{a}$, there exists a control $\Theta \in \Upsilon^{a}$ having the same cost.

Proposition 4.12. Assume that $C \doteq\left(\Omega, \mathcal{F}, P,\left\{\mathcal{F}_{t}\right\},\left\{u_{t}\right\},\left\{W_{t}\right\},\left\{x_{t}\right\}\right)$ is an element of $\mathfrak{C}^{a}$. Write $\Theta$ for $\left(\Omega, \mathcal{F}, P,\left\{\mathcal{F}_{\Phi_{t}}\right\},\left\{\theta_{t}\right\},\left\{V_{t}\right\},\left\{\Delta_{t}\right\}, \Gamma_{T}\right)$, where

$$
\theta_{t} \doteq \frac{u_{\Phi_{t}}}{1+\left|u_{\Phi_{t}}\right|}, \quad V_{t} \doteq \int_{0}^{\Phi_{t}} \sqrt{1+\left|u_{s}\right|} d W_{s}, \quad \Delta_{t} \doteq\left(\begin{array}{c}
\Phi_{t} \\
x_{\Phi_{t}}
\end{array}\right),
$$

and $\Phi_{t}$ (respectively, $\Gamma_{t}$ ) is defined by (47) (respectively, (46)). Then $\Theta$ belongs to $\Upsilon^{a}$ and

$$
\mathcal{M}[\Theta]=J[C] .
$$

Proof. From Proposition 1.1 in [20, Chapter V], $\left\{\mathcal{F}_{\Phi_{t}}\right\}$ defines an increasing and right continuous filtration which is complete. Then assertion (i) of Definition 4.1 is satisfied. Now, using Theorem 3.52 in [11], it follows that $\left\{\frac{u_{\Phi_{t}}}{1+\left|u_{\Phi_{t}}\right|}\right\}$ is an $\left\{\mathcal{F}_{\Phi_{t}}\right\}$ predictable process. Moreover, for all $t \in \mathbb{R}_{+}, \frac{u_{\Phi_{t}}}{1+\left|u_{\Phi_{t}}\right|} \in B_{1}(K)$. that

The process $\left\{N_{t} \doteq \int_{0}^{t} \sqrt{1+\left|u_{s}\right|} d W_{s}\right\}$ is an $\left\{\mathcal{F}_{t}\right\}$ continuous local martingale such

$$
\left(\forall t \in[0, T], \forall(i, j) \in \mathbb{N}_{n}^{2}\right) \quad\left\langle N^{i}, N^{i}\right\rangle_{t}=\Gamma_{t},\left\langle N^{i}, N^{j}\right\rangle_{t}=0 \quad(i \neq j) .
$$

Therefore, according to Theorem 4.13 in [12], $\left\{V_{t}=N_{\Phi_{t}}\right\}$ is an $\left\{\mathcal{F}_{\Phi_{t}}\right\}$ standard $m$-dimensional Brownian motion which gives item (iii) of Definition 4.1.

By Remark 2.9 and Theorem 2.33 in [6], the process $\left\{\Delta_{t}\right\}$ is adapted to $\left\{\mathcal{F}_{\Phi_{t}}\right\}$. Clearly, the process $\left\{\Delta_{t}\right\}$ is corlol. Consequently, $\left\{\Delta_{t}\right\}$ is progressively measurable with respect to $\left\{\mathcal{F}_{\Phi_{t}}\right\}$.

Using Proposition 1.1 in [20, Chapter V], $\Gamma_{T}$ is an $\left\{\mathcal{F}_{\Phi_{t}}\right\}$ stopping time. With (4), we have that $\Gamma_{T}=T+\int_{0}^{T}\left|u_{s}\right| d s \leq T+M$. Therefore, item (iv) of Definition 4.1 is satisfied.

Now let us show that the components of $\left\{\Delta_{t}\right\}$ satisfy (16) and (17) on $\left[0, \Gamma_{T}\right]$. Using (48) and the definition of $\{\theta\}$, we have

$$
\left(\forall t \in\left[0, \Gamma_{T}\right]\right) \quad \Phi_{t}=t-\int_{0}^{t}\left|\theta_{s}\right| d s .
$$

Therefore, the first component of the process $\left\{\Delta_{t}\right\}$ satisfies (16).

Now the process $\left\{x_{\Phi_{t}}\right\}$ satisfies (for all $t \in\left[0, \Gamma_{T}\right]$ )

$$
x_{\Phi_{t}} \doteq \zeta+\int_{0}^{\Phi_{t}} A\left(s, x_{s}\right) d s+\int_{0}^{\Phi_{t}} B\left(s, x_{s}\right) u_{s} d s+\int_{0}^{\Phi_{t}} D\left(s, x_{s}\right) d W_{s} .
$$

Since $\left\{\Gamma_{t}\right\}$ is continuous, we can use Proposition 1.4 in [20, Chapter V] and Lemma 4.10 in order to obtain that

$$
\begin{aligned}
\left(\forall t \in\left[0, \Gamma_{T}\right]\right) \quad \int_{0}^{\Phi_{t}} A\left(s, x_{s}\right) d s & =\int_{0}^{t} A\left(\Phi_{s}, x_{\Phi_{s}}\right) d \Phi_{s} \\
& =\int_{0}^{t} A\left(\Phi_{s}, x_{\Phi_{s}}\right)\left(1-\left|\theta_{s}\right|\right) d s .
\end{aligned}
$$

We can repeat the same argument to show that

$$
\left(\forall t \in\left[0, \Gamma_{T}\right]\right) \quad \int_{0}^{\Phi_{t}} B\left(s, x_{s}\right) u_{s} d s=\int_{0}^{t} B\left(\Phi_{s}, x_{\Phi_{s}}\right) \theta_{s} d s .
$$


Moreover,

$$
\begin{aligned}
\int_{0}^{\Phi_{t}} D\left(s, x_{s}\right) d W_{s} & =\int_{0}^{\Phi_{t}} D\left(s, x_{s}\right) \frac{1}{\sqrt{1+\left|u_{s}\right|}} d N_{s} \\
& =\int_{0}^{t} D\left(\Phi_{s}, x_{\Phi_{s}}\right) \sqrt{1-\left|\theta_{s}\right|} d V_{s}
\end{aligned}
$$

where the last equality is obtained by using Proposition 4.8 in [12].

Combining (53)-(56), we obtain that the process $\left\{x_{\Phi_{t}}\right\}$ satisfies

$$
\begin{aligned}
\left(\forall t \in\left[0, \Gamma_{T}\right]\right) \quad x_{\Phi_{t}}=\zeta & +\int_{0}^{t}\left(1-\left|\theta_{s}\right|\right) A\left(\Phi_{s}, x_{\Phi_{s}}\right) d s+\int_{0}^{t} B\left(\Phi_{s}, x_{\Phi_{s}}\right) \theta_{s} d s \\
& +\int_{0}^{t} \sqrt{1-\left|\theta_{s}\right|} D\left(\Phi_{s}, x_{\Phi_{s}}\right) d V_{s} .
\end{aligned}
$$

Therefore, assertion (iv) of Definition 4.1 is satisfied for the process $\left\{\Delta_{t}\right\}$ (see (49) for its definition). Finally, it follows that $\Theta \in \bar{\Upsilon}$. However, we have shown that $\left\{\theta_{t}\right\}$ is a $B_{1}(K)$-valued process. Consequently, $\Theta \in \Upsilon$.

Now the cost corresponding to $\Theta$ is given by $\mathcal{M}(\Theta)=E_{P}\left[g\left(x_{\Phi_{\Gamma_{T}}}\right)+G\left(\Phi_{\Gamma_{T}}\right)\right]$.

However, $\Phi_{\Gamma_{T}}=T$ (see item (i) of Lemma 4.10). Therefore, we have $\mathcal{M}[\Theta]=$ $E_{P}\left[g\left(x_{T}\right)\right]=J[C]<\infty$, implying that $\Theta \in \Upsilon^{a}$.

The proof of the following lemma is similar to that of Lemma 4.10. Therefore, it is omitted.

Lemma 4.13. Let $\left(\Omega, \mathcal{F}, P,\left\{\mathcal{G}_{t}\right\},\left\{\theta_{t}\right\},\left\{V_{t}\right\},\left\{\Lambda_{t}=\left(\eta_{t}, \xi_{t}^{\prime}\right)^{\prime}\right\}, \gamma\right)$ be an element of $\Upsilon$, and let $\left\{\psi_{t}\right\}$ be the right inverse of $\eta$ :

$$
\psi_{t} \doteq \inf \left\{s \geq 0: \eta_{s}>t\right\}
$$

The process $\left\{\psi_{t}\right\}$ is a continuous time-change satisfying the following properties:

(i) $\left(\forall t \in \mathbb{R}_{+}\right) \psi_{\eta_{t}}=t$ and $\eta_{\psi_{t}}=t$.

(ii) $\left(\forall t \in \mathbb{R}_{+}\right) \psi_{t}=\int_{0}^{t} \frac{1}{1-\left|\theta_{\psi_{s}}\right|} d s$.

Conversely to Proposition 4.12, we show that, for any control $\Psi \in \Upsilon^{a}$, there exists a control $S \in \mathfrak{C}^{a}$ having the same cost. The ideas to show this result are the same as the one used in the proof of Proposition 4.12. Consequently, this result is quoted without proof.

Proposition 4.14. Let $\Psi \doteq\left(\Omega, \mathcal{F}, P,\left\{\mathcal{G}_{t}\right\},\left\{\theta_{t}\right\},\left\{V_{t}\right\},\left\{\Lambda_{t}=\left(\eta_{t}, \xi_{t}^{\prime}\right)^{\prime}\right\}, \gamma\right)$ be an element of $\Upsilon^{a}$. Write $S$ for $\left(\Omega, \mathcal{F}, P,\left\{\mathcal{G}_{\psi_{t}}\right\},\left\{u_{t}\right\},\left\{W_{t}\right\},\left\{\xi_{\psi_{t}}\right\}\right)$, where

$$
u_{t} \doteq \frac{\theta_{\psi_{t}}}{1-\left|\theta_{\psi_{t}}\right|}, \quad W_{t} \doteq \int_{0}^{\psi_{t}} \sqrt{1-\left|\theta_{s}\right|} d V_{s}
$$

and $\left\{\Psi_{t}\right\}$ is defined in (58).

Then $S$ belongs to $\mathfrak{C}^{a}$, and

$$
J[S]=\mathcal{M}[\Psi] .
$$

Now we obtain the following result.

THEOREM 4.15. The following property holds:

$$
\inf _{C \in \mathfrak{C}^{a}} J[C]=\inf _{\Psi \in \Upsilon^{a}} \mathcal{M}[\Psi] .
$$


Proof. The result is an immediate consequence of Propositions 4.12 and 4.14.

Finally, we derive an important characterization of $\inf _{C \in \mathfrak{C}^{a}} J[C]$.

Corollary 4.16. Let $\Theta^{*} \in \bar{\Upsilon}^{a}$ be the optimal control for the auxiliary control problem. Then

$$
\inf _{C \in \mathfrak{C}^{a}} J[C]=\mathcal{M}\left[\Theta^{*}\right]
$$

Proof. It is a straightforward combination of Theorems 4.9 and 4.15 .

REMARK 4.17. Let us denote by $\Theta^{*} \doteq\left(\Omega, \mathcal{F}, P,\left\{\mathcal{G}_{t}\right\},\left\{\theta_{t}\right\},\left\{V_{t}\right\},\left\{\left(\eta_{t}, \xi_{t}^{\prime}\right)^{\prime}\right\}, \gamma\right)$ the optimal control in $\bar{\Upsilon}^{a}$. There is no loss of generality to assume that

$$
\inf \left\{s: \eta_{s}>T\right\}=\gamma .
$$

Indeed, if this is not the case, let $\widetilde{\Theta}$ be the control defined by

$$
\begin{aligned}
& \widetilde{\Theta} \doteq\left(\Omega, \mathcal{F}, P,\left\{\mathcal{G}_{t}\right\},\left\{\widetilde{\theta}_{t}\right\},\left\{V_{t}\right\},\left\{\left(\widetilde{\eta}_{t}, \widetilde{\xi}_{t}^{\prime}\right)^{\prime}\right\}, \gamma\right), \\
& \widetilde{\theta}_{t} \doteq \theta_{t} I_{[0, \gamma]}, \\
& \widetilde{\eta}_{t} \doteq t-\int_{0}^{t}\left|\widetilde{\theta}_{s}\right| d s, \\
& \widetilde{\xi}_{t} \doteq \zeta+\int_{0}^{t}\left(1-\left|\widetilde{\theta}_{s}\right|\right) A\left(\widetilde{\eta}_{s}, \widetilde{\xi}_{s}\right) d s+\int_{0}^{t} B\left(\widetilde{\eta}_{s}, \widetilde{\xi}_{s}\right) \widetilde{\theta}_{s} d s \\
& \quad+\int_{0}^{t} \sqrt{1-\left|\widetilde{\theta}_{s}\right|} D\left(\widetilde{\eta}_{s}, \widetilde{\xi}_{s}\right) d V_{s} .
\end{aligned}
$$

Clearly, $\widetilde{\Theta} \in \Upsilon^{a}$ and $\inf \left\{s: \widetilde{\eta}_{s}>T\right\}=\gamma$. Moreover, it is easy to check that (for all $t \in[0, \gamma]) \widetilde{\theta}_{t}=\theta_{t}, \widetilde{\eta}_{t}=\eta_{t}$, and $\widetilde{\xi}_{t}=\xi_{t}$. Therefore,

$$
\mathcal{M}(\widetilde{\Theta})=\mathcal{M}\left(\Theta^{*}\right)=\min _{\Psi \in \bar{\Upsilon}^{a}} \mathcal{M}[\Psi] .
$$

5. Existence of an optimal generalized control. In this section, we obtain the last characterization of $\inf _{C \in \mathfrak{C}^{a}} J[C]$ in terms of an optimal generalized control.

TheOREM 5.1. There exists a generalized control $C^{{ }^{*}} \in \overline{\mathfrak{C}}^{a}$ such that

$$
\begin{aligned}
\inf _{C \in \mathfrak{C}^{a}} J[C] & =J\left[C^{g *}\right] \\
& =\min _{C^{g} \in \overline{\mathfrak{C}}^{a}} J\left[C^{g}\right] .
\end{aligned}
$$

Proof. Let us denote by

$$
\Theta^{*} \doteq\left(\Omega, \mathcal{F}, P,\left\{\mathcal{G}_{t}\right\},\left\{\theta_{t}\right\},\left\{V_{t}\right\},\left\{\left(\eta_{t}, \xi_{t}^{\prime}\right)^{\prime}\right\}, \gamma\right)
$$

the optimal control in $\bar{\Upsilon}^{a}$.

Define

$$
\begin{aligned}
\psi_{t} & \doteq \inf \left\{s: \eta_{s}>t\right\}, \\
X_{t} & \doteq \xi_{\psi_{t}}, \\
U_{t} & \doteq \int_{0}^{\psi_{t}} \theta_{s} d s, \\
W_{t} & \doteq \int_{0}^{\psi_{t}} \sqrt{1-\left|\theta_{s}\right|} d V_{s} .
\end{aligned}
$$


On the probability space $(\Omega, \mathcal{F}, P),\left\{\psi_{t}\right\}$ is a time-change (see Proposition 1.1 in [20, Chapter V]). Moreover, $\left\{\mathcal{G}_{\psi_{t}}\right\}$ defines a right continuous complete filtration. Therefore, the processes $\left\{X_{t}\right\},\left\{Y_{t}\right\}$, and $\left\{U_{t}\right\}$ are $\left\{\mathcal{G}_{\psi_{t}}\right\}$ progressively measurable (see Theorem T57, page 105 in [14]). Since $\left\{\xi_{t}\right\}$ is a continuous process, $\left\{X_{t}\right\},\left\{Y_{t}\right\}$, and $\left\{U_{t}\right\}$ are corlol. Moreover, since $K$ is a separable metric space satisfying assumption (A.3), it is easy to obtain that $\left\{U_{t}\right\}$ is a $K$-valued process and $U_{t}-U_{s} \in K$ for $t \geq s$.

According to Theorem 4.13 in $[12],\left\{W_{t}\right\}$ is a $\left\{\mathcal{G}_{\psi_{t}}\right\}$ standard $m$-dimensional Brownian motion.

Now, using Theorem 6.46 in [6], there exists a sequence $\left\{\tau_{n}\right\}$ of stopping times which exhausts the jumps of $\left\{\psi_{t}\right\}$. Clearly, we have

$$
\bigcup_{n=1}^{\infty} \llbracket \psi_{\tau_{n}-}, \psi_{\tau_{n}} \rrbracket \subset\left\{(t, \omega) \in \mathbb{R}_{+} \times \Omega:\left|\theta_{t}\right|=1\right\} .
$$

Define

$$
\mathcal{D} \doteq\left\{(t, \omega) \in \mathbb{R}_{+} \times \Omega:\left|\theta_{t}\right|=1\right\}-\bigcup_{n=1}^{\infty} \llbracket \psi_{\tau_{n}-}, \psi_{\tau_{n}} \rrbracket
$$

Consequently,

$$
\begin{aligned}
(\forall t \in[0, T]) \quad U_{t} & =\int_{0}^{\psi_{t}} I_{\left\{\left|\theta_{s}\right|<1\right\}} \theta_{s} d s+\int_{0}^{\psi_{t}} I_{\left\{\left|\theta_{s}\right|=1\right\}} \theta_{s} d s \\
& =\int_{0}^{\psi_{t}}\left[I_{\left\{\left|\theta_{s}\right|<1\right\}}+I_{\mathcal{D}}\right] \theta_{s} d s+\sum_{n \in \mathbb{N}} \int_{\psi_{\tau_{n}-}}^{\psi_{\tau_{n}}} \theta_{s} d s I_{\left[\tau_{n}, \infty \llbracket\right.} .
\end{aligned}
$$

For $(t, \omega) \in \cup_{n=1}^{\infty} \llbracket \psi_{\tau_{n}-}, \psi_{\tau_{n}} \rrbracket$, we have $I_{\left\{\left|\theta_{t}\right|<1\right\}}(\omega)+I_{\mathcal{D}}(t, \omega)=0$.

Therefore, $\left\{\int_{0}^{t}\left[I_{\left\{\left|\theta_{s}\right|<1\right\}}+I_{\mathcal{D}}\right] \theta_{s} d s\right\}$ is a $\left\{\psi_{t}\right\}$ continuous process. Consequently, the decomposition of the process $\left\{U_{t}\right\}$ is given by

$$
\begin{aligned}
U_{t}^{c} & =\int_{0}^{\psi_{t}}\left[I_{\left\{\left|\theta_{s}\right|<1\right\}}+I_{\mathcal{D}}\right] \theta_{s} d s, \\
U_{t}^{d} & =\sum_{n \in \mathbb{N}} \int_{\psi_{\tau_{n}-}}^{\psi_{\tau_{n}}} \theta_{s} d s I_{\llbracket \tau_{n}, \infty \llbracket \cdot}
\end{aligned}
$$

From Lemma 1.37 in [11], we have

$$
\left(\forall t \in \mathbb{R}_{+}\right) \quad \eta_{\psi_{t}}=t .
$$

Moreover, using Proposition 4.8 in [12], it follows that

$$
(\forall t \in[0, T]) \quad \int_{0}^{\psi_{t}} \sqrt{1-\left|\theta_{s}\right|} D\left(\eta_{s}, \xi_{s}\right) d V_{s}=\int_{0}^{t} D\left(s, \xi_{\psi_{s}}\right) d W_{s} .
$$

Note that $\left\{\eta_{t}\right\}$ is a $\left\{\psi_{t}\right\}$ continuous process. Moreover, $\left\{\eta_{t}\right\}$ is a process of finite variation because it is absolutely continuous. Therefore, using Proposition 1.4 in [20, Chapter V] and (67), we obtain that

$$
\begin{aligned}
(\forall t \in[0, T)) \quad \int_{0}^{\psi_{t}}\left(1-\left|\theta_{s}\right|\right) A\left(\eta_{s}, \xi_{s}\right) d s & =\int_{0}^{\psi_{t}} A\left(\eta_{s}, \xi_{s}\right) d \eta_{s} \\
& =\int_{0}^{t} A\left(s, \xi_{\psi_{s}}\right) d s .
\end{aligned}
$$


Again, using the fact that $\left\{\int_{0}^{t}\left[I_{\left\{\left|\theta_{s}\right|<1\right\}}+I_{\mathcal{D}}\right] \theta_{s} d s\right\}$ is a $\left\{\psi_{t}\right\}$ continuous process and Proposition 1.4 in [20, Chapter V], we have

$$
\begin{aligned}
\int_{0}^{\psi_{t}} B\left(\eta_{s}, \xi_{s}\right) \theta_{s} d s= & \int_{0}^{\psi_{t}} B\left(\eta_{s}, \xi_{s}\right)\left[I_{\left\{\left|\theta_{s}\right|<1\right\}}+I_{\mathcal{D}}\right] \theta_{s} d s \\
& +\sum_{n \in \mathbb{N}} \int_{\psi_{\tau_{n}-}}^{\psi_{\tau_{n}}} B\left(\eta_{s}, \xi_{s}\right) \theta_{s} d s I_{\llbracket \tau_{n}, \infty \llbracket} \\
= & \int_{0}^{t} B\left(s, \xi_{\psi_{s}}\right) d U_{s}^{c}+\sum_{n \in \mathbb{N}} \int_{\psi_{\tau_{n}-}}^{\psi_{\tau_{n}}} B\left(\eta_{s}, \xi_{s}\right) \theta_{s} d s I_{\left[\tau_{n}, \infty[\right.} .
\end{aligned}
$$

It follows that the process $\left\{X_{t}\right\}$ satisfies the following equation:

$$
\begin{aligned}
(\forall t \in[0, T]) \quad X_{t}=\zeta & +\int_{0}^{t} A\left(s, X_{s}\right) d s+\int_{0}^{t} B\left(s, X_{s}\right) d U_{s}^{c}+\int_{0}^{t} D\left(s, X_{s}\right) d W_{s} \\
& +\sum_{n \in \mathbb{N}} \Delta X_{\tau_{n}} I_{\left[\tau_{n}, \infty \mathbb{I}\right.}
\end{aligned}
$$

where

$$
\Delta X_{\tau_{n}} \doteq \int_{\psi_{\tau_{n}-}}^{\psi_{\tau_{n}}} B\left(\eta_{s}, \xi_{s}\right) \theta_{s} d s
$$

According to Proposition 4.8, there exists a sequence $\left\{\Psi^{n}\right\}$ such that $\Psi^{n} \in \Upsilon^{a}$ for all $n \in \mathbb{N}$. Write

$$
\psi_{t}^{n} \doteq \inf \left\{s: \eta_{s}^{n}>t\right\}
$$

From Proposition 4.6, it follows that $\left\{\psi_{t}^{n}\right\}$ is a continuous, strictly increasing process and such that

$$
\left(\forall t \in\left[0, \frac{n T}{n+1}\right)\right) \quad \psi_{t}^{n} \leq \psi_{t}^{n+1} \leq \psi_{t} .
$$

Therefore, for $t$ in $[0, T)), \bar{\psi}_{t} \doteq \lim _{n \rightarrow \infty} \psi_{t}^{n}$ exists. Again, using (70), this limit is lower semicontinuous and increasing on $[0, T)$.

Using similar arguments as in the proof of Lemma 4.5, it can be shown easily that

$$
(\forall t \in[0, T)) \quad \eta_{\bar{\psi}_{t}}=t .
$$

Combining (67) with (71), we obtain that

$$
\left(\forall t \in \mathbb{R}_{+}\right) \quad \sum_{n} I_{\rrbracket} \tau_{n} \wedge T, \tau_{n+1} \wedge T\left[\psi_{t}=\sum_{n} I_{\rrbracket} \wedge \tau_{n} \wedge T, \tau_{n+1} \wedge T\left[\bar{\psi}_{t} .\right.\right.
$$

However, recalling that $\left\{\bar{\psi}_{t}\right\}$ is a lower semicontinuous, increasing process and $\left\{\psi_{t}\right\}$ is corlol, it follows that $\left\{\bar{\psi}_{t}\right\}$ is collor and

$$
(\forall t \in[0, T)) \quad \psi_{t}=\bar{\psi}_{t+} .
$$

There is no loss of generality to assume that $\lim _{t \rightarrow 0 t<0} \psi_{t}=0$, and so

$$
(\forall t \in[0, T)) \quad \bar{\psi}_{t}=\psi_{t-} .
$$


Moreover, since $\psi^{n} \in \Upsilon^{a}$, we have that $\eta_{\gamma^{n}}^{n}=T$. Recalling that $\left\{\eta_{t}^{n}\right\}$ is strictly increasing and continuous, we obtain that $\psi_{T}^{n}=\gamma^{n}$. Note that $\psi_{T}=\gamma$ (see Remark 4.17). Using Lemma 4.7, it follows that

$$
\bar{\psi}_{T} \doteq \lim _{n \rightarrow \infty} \psi_{T}^{n}=\psi_{T}=\gamma, \quad P-\text { a.s. }
$$

Using (73) and the fact that $\left\{\psi_{t}\right\}$ is an increasing process, we have that, for all $t \in[0, T), \bar{\psi}_{t} \leq \psi_{T-} \leq \psi_{T}=\bar{\psi}_{T}$. Consequently, $\left\{\bar{\psi}_{t}\right\}$ is increasing.

However, using similar arguments as in the proof of Proposition 4.8 (see (42)), it can be shown easily that there exists a subsequence, still denoted by $n$, such that (for all $t \in[0, T]$ )

(75) $\lim _{n \rightarrow \infty} \xi_{\psi_{t}^{n}}^{n}=\xi_{\bar{\psi}_{t}}, \quad P$ - a.s., and $\lim _{n \rightarrow \infty} \int_{0}^{\psi_{t}^{n}}\left|\theta_{s}^{n}\right| d s=\int_{0}^{\bar{\psi}_{t}}\left|\theta_{s}\right| d s, \quad P-$ a.s.

Define

$$
C^{n}=\left(\Omega, \mathcal{F}, P,\left\{\mathcal{G}_{\psi_{t}^{n}}\right\},\left\{\frac{\theta_{\psi_{t}^{n}}^{n}}{1-\left|\theta_{\psi_{t}^{n}}^{n}\right|}\right\},\left\{\int_{0}^{\psi_{t}^{n}} \sqrt{1-\left|\theta_{s}^{n}\right|} d V_{s}\right\},\left\{\left(\psi_{t}^{n}-t, \xi_{\psi_{t}^{n}}^{n}\right)^{\prime}\right\}\right) .
$$

Since $\Psi^{n} \in \Upsilon^{a}$ for all $n \in \mathbb{N}$ and using Proposition 4.14, it follows that $C^{n} \in \mathfrak{C}^{a}$ for all $n \in \mathbb{N}$.

Using the fact that $\left\{\xi_{t}\right\}$ is continuous, (72), and (75), we obtain that

$$
(\forall t \in[0, T)) \quad X_{t}=\lim _{\substack{s \rightarrow t \\ s>t}} \lim _{n \rightarrow \infty} \xi_{\psi_{s}^{n}}^{n}, \quad P-\text { a.s. }
$$

Moreover, using (74) and (75),

$$
X_{T}=\xi_{\psi_{T}}=\xi_{\bar{\psi}_{T}}=\lim _{n \rightarrow \infty} \xi_{\psi_{T}^{n}}^{n}, \quad P-\text { a.s. }
$$

From the definition of $\left\{\psi_{t}^{n}\right\}$ and since $\Psi^{n} \in \Upsilon^{a}$, we can use Lemma 4.13 in order to obtain

$$
\begin{aligned}
(\forall t \in[0, T]) \quad \psi_{t}^{n}-t & =\int_{0}^{t} \frac{\left|\theta_{\psi_{s}^{n}}^{n}\right|}{1-\left|\theta_{\psi_{s}^{n}}^{n}\right|} d s \\
& =\int_{0}^{t}\left|\theta_{\psi_{s}^{n}}^{n}\right| d \psi_{s}^{n} .
\end{aligned}
$$

Using Proposition 1.4 in [20, Chapter V] and the fact that $\left\{\psi_{t}^{n}\right\}$ is a continuous process, we have

$$
(\forall t \in[0, T]) \quad \psi_{t}^{n}-t=\int_{0}^{\psi_{t}^{n}}\left|\theta_{s}^{n}\right| d s .
$$

Therefore, combining (74), (75), and (77) yields

$$
\int_{0}^{\psi_{T}}\left|\theta_{s}\right| d s=\gamma-T
$$

However,

$$
\underset{[0, T]}{\operatorname{Var}}\left[U_{t}\right] \leq \operatorname{Var}_{[0, T]}\left[\int_{0}^{\psi_{t}}\left|\theta_{s}\right| d s\right]=\int_{0}^{\psi_{T}}\left|\theta_{s}\right| d s
$$


Consequently, using (15), it follows that

$$
\operatorname{Var}_{[0, T]}\left[U_{t}\right] \leq M
$$

Finally, the generalized control $C^{g *}$ defined by

$$
C^{g *} \doteq\left(\Omega, \mathcal{F}, P,\left\{\mathcal{F}_{t}\right\},\left\{U_{t}\right\},\left\{W_{t}\right\},\left\{X_{t}\right\}\right)
$$

is an element of $\overline{\mathfrak{C}}^{a}$.

However, by hypothesis, $\inf _{C \in \mathfrak{C}^{a}} J[C]=E_{P}\left[g\left(\xi_{\gamma}\right)\right]$, and so we obtain with (76)

$$
\inf _{C \in \mathfrak{C}^{a}} J[C]=E_{P}\left[g\left(X_{T}\right)\right]=J\left[C^{g *}\right] .
$$

Now, using Proposition 3.2, we obtain the result.

Appendix. In this section, we prove some technical results.

Proof of Lemma 2.2. Let us consider $R>|\zeta|$ and define $\tau_{R} \doteq \inf \left\{t:\left|x_{t}\right| \geq R\right\}$. Clearly, the process $\left\{x_{t \wedge \tau_{R}}\right\}$ is solution of the following equation:

$$
\begin{aligned}
x_{t \wedge \tau_{R}} \doteq \zeta & +\int_{0}^{t} A\left(s, x_{s \wedge \tau_{R}}\right) I_{\left\{s \leq \tau_{R}\right\}} d s+\int_{0}^{t} B\left(s, x_{s \wedge \tau_{R}}\right) u_{s} I_{\left\{s \leq \tau_{R}\right\}} d s \\
& +\int_{0}^{t} D\left(s, x_{s \wedge \tau_{R}}\right) I_{\left\{s \leq \tau_{R}\right\}} d W_{s} .
\end{aligned}
$$

Using (A.1) and (4), it follows that

$$
\begin{aligned}
\left|x_{t \wedge \tau_{R}}\right| \leq|\zeta| & +L_{1}(T+M)+\left|\int_{0}^{t} D\left(s, x_{s \wedge \tau_{R}}\right) I_{\left\{s \leq \tau_{R}\right\}} d W_{s}\right| \\
& +\int_{0}^{t}\left|x_{s \wedge \tau_{R}}\right| L_{1}\left(1+\left|u_{t}\right|\right) d s .
\end{aligned}
$$

Using Gronwall's lemma, we obtain that

$$
\left|x_{t \wedge \tau_{R}}\right| \leq M_{1}+M_{2} \sup _{s \leq t}\left|\int_{0}^{s} D\left(s, x_{s \wedge \tau_{R}}\right) I_{\left\{s \leq \tau_{R}\right\}} d W_{s}\right|,
$$

where $M_{1}$ and $M_{2}$ are two constants. Using Theorem 6.5, page 87 in [7], assumption (A.1), and Gronwall's lemma, we finally have that

$$
E_{P}\left[\sup _{t \leq T}\left|x_{t \wedge \tau_{R}}\right|^{2 q}\right] \leq M
$$

for a constant $M$.

Due to the continuity of $\left\{x_{t}\right\}, \tau_{R} \rightarrow \infty$ as $R \rightarrow \infty$. Therefore, using Fatou's lemma and the previous equation, the result follows.

Lemma A.1. Suppose $(\Omega, \mathcal{F}, P)$ is a probability space with a filtration $\left\{\mathcal{G}_{t}\right\}$ and $\left\{V_{t}\right\}$ is a $\left\{\mathcal{G}_{t}\right\}$ standard Brownian motion. Then $\left\{V_{t}\right\}$ is a $\left\{\mathcal{G}_{t}^{q}\right\}$ standard Brownian motion on the probability space $\left(\Omega, \mathcal{F}^{q}, \bar{P}\right)$, where

$$
\begin{aligned}
& \mathcal{F}^{q} \doteq\{A \subset \Omega:(\exists B \in \mathcal{F}) \text { such that } A \triangle B \in \mathcal{N}\} \\
& \mathcal{G}_{t}^{q} \doteq\left\{A \subset \Omega:\left(\exists B \in \mathcal{G}_{t}\right) \text { such that } A \triangle B \in \mathcal{N}\right\} \\
& \mathcal{N} \doteq\{A \subset \Omega:(\exists B \in \mathcal{F}) \text { such that } A \subset B \text { and } P(B)=0\}
\end{aligned}
$$


and the probability $\bar{P}$ is defined by (for all $\left.A \in \mathcal{F}^{q}\right) \bar{P}(A)=P(B)$, where $B \in \mathcal{F}$ and $A \triangle B \in \mathcal{N}$.

Proof. From the definition of $\mathcal{G}_{s}^{q}$ and $\mathcal{N}$, it follows that

$$
\left(\forall s>0, \quad \forall A \in \mathcal{G}_{s}^{q}\right) \quad\left(\exists(B, N) \in \mathcal{G}_{s} \times \mathcal{N}\right) \quad \text { such that } \quad A=B+N .
$$

Therefore, for all $t>s \geq 0$ and for all $A \in \mathcal{G}_{s}^{q}$ we have

$$
\int_{A} \exp \left[i u^{\prime}\left(V_{t}-V_{s}\right)\right] d \bar{P}=\exp -\frac{|u|^{2}(t-s)}{2} P(B) .
$$

Moreover, $P(B)=\bar{P}(A)$, and, using (78), we obtain

$$
E_{\bar{P}}\left[\exp \left[i u^{\prime}\left(V_{t}-V_{s}\right)\right] \mid \mathcal{G}_{s}^{q}\right]=\exp -\frac{|u|^{2}(t-s)}{2},
$$

which gives the result.

Lemma A.2. Suppose $(\Omega, \mathcal{F}, P)$ is a complete probability space with a complete filtration $\left\{\mathcal{G}_{t}\right\}$ and $\left\{V_{t}\right\}$ is a $\left\{\mathcal{G}_{t}\right\}$ standard Brownian motion. Then $\left\{V_{t}\right\}$ is a $\left\{\mathcal{G}_{t+}\right\}$ standard Brownian motion.

Proof. For all $t>s \geq 0$ and $0<\epsilon<t-s$ we have

$$
E_{P}\left[\exp \left[i u^{\prime}\left(V_{t}-V_{s+\epsilon}\right)\right] \mid \mathcal{G}_{s+\epsilon}\right]=\exp -\frac{|u|^{2}(t-s-\epsilon)}{2} .
$$

Since $\mathcal{G}_{s+}=\bigcap_{\epsilon>0} \mathcal{G}_{s+\epsilon}$, we obtain that

$$
\left(\forall A \in \mathcal{G}_{s+}\right) \quad \lim _{\substack{\epsilon \rightarrow 0 \\ \epsilon>0}} \int_{A} \exp \left[i u^{\prime}\left(V_{t}-V_{s+\epsilon}\right)\right] d P=\exp -\frac{|u|^{2}(t-s)}{2} P(A) .
$$

By using the bounded convergence theorem and the fact that the $\left\{V_{t}\right\}$ is a continuous process, we have

$$
E_{P}\left[\exp \left[i u^{\prime}\left(V_{t}-V_{s}\right)\right] \mid \mathcal{G}_{s+}\right]=\exp -\frac{|u|^{2}(t-s)}{2},
$$

and the result follows.

Lemma A.3. Assume that $\left\{\theta_{t}\right\}$ is a $\bar{B}_{1}(K)$-valued, $\left\{\mathcal{G}_{t}\right\}$ progressively measurable process. Then (16) and (17) have a unique solution such that

$$
(\forall q \in \mathbb{N}) \quad E_{P}\left[\sup _{t \in[0, \gamma]}\left|\xi_{t}\right|^{2 q}\right]<\infty
$$

Moreover, there exist a $\bar{B}_{1}(K)$-valued, $\left\{\mathcal{G}_{t}\right\}$-predictable process $\left\{\bar{\theta}_{t}\right\}$ such that

$$
\bar{\theta}=\theta, \quad \lambda \otimes P-\text { a.e. },
$$

and the process $\left\{\bar{\xi}_{t}\right\}$ solution of the following stochastic differential equation:

$$
\bar{\xi}_{t} \doteq \zeta+\int_{0}^{t}\left(1-\left|\bar{\theta}_{s}\right|\right) A\left(\bar{\eta}_{s}, \bar{\xi}_{s}\right) d s+\int_{0}^{t} B\left(\bar{\eta}_{s}, \bar{\xi}_{s}\right) \bar{\theta}_{s} d s+\int_{0}^{t} \sqrt{1-\left|\bar{\theta}_{s}\right|} D\left(\bar{\eta}_{s}, \bar{\xi}_{s}\right) d V_{s}
$$

where $\bar{\eta}_{t}=t-\int_{0}^{t}\left|\bar{\theta}_{s}\right| d s$ is indistinguishable from $\left\{\xi_{t}\right\}$. 
Proof. Using (A.1) and Theorem 7, page 197 in [19], the existence and the uniqueness of the solution are straightforward. The conditions of Corollary 10, page 85 in [13] are satisfied, and the inequality (79) follows.

By hypothesis, the process $\theta$ is progressively measurable with respect to $\left\{\mathcal{G}_{t}\right\}$. Using Theorem 3.7 in [3], it follows that the function $\theta: \mathbb{R}_{+} \times \Omega \rightarrow K$ is $\mathcal{P}^{*}$ measurable, where

$$
\mathcal{P}^{*} \doteq\left\{A \in \mathcal{B}\left(\mathbb{R}_{+}\right) \otimes \mathcal{F}: A \triangle B \in \mathcal{N} \text { for some } B \in \mathcal{P}\right\},
$$

$\mathcal{P}$ denoting the predictable $\sigma$-field and $\mathcal{N} \doteq\left\{N \in \mathcal{B}\left(\mathbb{R}_{+}\right) \otimes \mathcal{F}: \lambda \otimes P(N)=0\right\}$. Since $\bar{B}_{1}(K)$ is a locally compact separable metric space, we can use the lemma and its associated remark $\left[2\right.$, pp. 59-60] to obtain the existence of a $\bar{B}_{1}(K)$-valued, $\left\{\mathcal{G}_{t}\right\}$ predictable process $\left\{\bar{\theta}_{t}\right\}$ satisfying $(80)$.

Consequently,

$$
\eta_{t}=t-\int_{0}^{t}\left|\bar{\theta}_{s}\right| d s
$$

Moreover, since $\left\{\bar{\eta}_{t}\right\}$ and $\left\{\eta_{t}\right\}$ are continuous, they are indistinguishable processes. Combining (81), (8), and (A.1), we obtain that $\int_{[0, T+M] \times \Omega}\left|B\left(., \bar{x}_{.}\right) u.\right| \lambda \otimes P<\infty$, so

$$
(\forall t \in[0, \gamma]) \quad \int_{0}^{t} B\left(\bar{\eta}_{s}, \bar{\xi}_{s}\right) \bar{\theta}_{s} d s=\int_{0}^{t} B\left(\eta_{s}, \bar{\xi}_{s}\right) \theta_{s} d s
$$

by using Fubini's theorem.

Similarly, we have that

$$
(\forall t \in[0, \gamma]) \quad \int_{0}^{t}\left(1-\left|\bar{\theta}_{s}\right|\right) A\left(\bar{\eta}_{s}, \bar{\xi}_{s}\right) d s=\int_{0}^{t}\left(1-\left|\theta_{s}\right|\right) A\left(\eta_{s}, \bar{\xi}_{s}\right) d s
$$

and

$$
(\forall t \in[0, \gamma]) \quad \int_{0}^{t}\left(1-\left|\bar{\theta}_{s}\right|\right)\left|D\left(\bar{\eta}_{s}, \bar{\xi}_{s}\right)\right|^{2} d s=\int_{0}^{t}\left(1-\left|\theta_{s}\right|\right)\left|D\left(\eta_{s}, \bar{\xi}_{s}\right)\right|^{2} d s
$$

Consequently, we obtain that

$$
(\forall t \in[0, \gamma]) \quad \int_{0}^{t} \sqrt{1-\mid \bar{\theta}_{s}} D\left(\bar{\eta}_{s}, \bar{\xi}_{s}\right) d s=\int_{0}^{t} \sqrt{1-\left|\theta_{s}\right|} D\left(\eta_{s}, \bar{\xi}_{s}\right) d s
$$

which implies that $\left\{\bar{\xi}_{t}\right\}$ satisfies (17).

By the uniqueness of the solution of (17), $\xi_{t}=\bar{\xi}_{t}, P-$ a.s., for all $t$ in $[0, \gamma]$. However, $\left\{\xi_{t}\right\}$ and $\left\{\bar{\xi}_{t}\right\}$ are continuous processes, so they are indistinguishable.

Proof of Lemma 4.5. Clearly, $\nu$ and $\nu^{n}$ are $\left\{\mathcal{G}_{t}\right\}$ stopping times (for all $n \in \mathbb{N}$ ). Since $\Psi \in \bar{\Upsilon}^{a}$, we have that $E_{P}\left[G\left(\eta_{\gamma}\right)\right]<\infty$, implying that $\eta_{\gamma}=T$. With (15) and the definition of $\nu$, we obtain that

$$
\nu \leq \gamma \leq T+M
$$

Note that

$$
\nu^{n} \leq \inf \left\{t \geq 0: t-\int_{0}^{t} \frac{n+1}{n+2}\left|\theta_{s}\right| d s \geq \frac{n T}{n+1}\right\}
$$


Since the process $\left\{t-\int_{0}^{t} \frac{n}{n+1}\left|\theta_{s}\right| d s\right\}$ is continuous and strictly increasing, we have $\nu^{n}<\nu^{n+1}$. Similarly, it can be shown that

$$
\nu^{n}<\nu
$$

Therefore, the sequence $\left\{\nu^{n}\right\}$ converges almost surely to a limit labeled $\bar{\nu}$ such that

$$
\bar{\nu} \leq \nu
$$

By definition of $\nu^{n}$, we have

$$
\nu^{n}-\int_{0}^{\nu^{n}} \frac{n}{n+1}\left|\theta_{s}\right| d s=\frac{n T}{n+1},
$$

and letting $n \rightarrow \infty$, we obtain

$$
\eta_{\bar{\nu}}=T
$$

From the definition of $\nu$, we have $\bar{\nu} \geq \nu$. However, with (84) we obtain that $\bar{\nu}=\nu$, and so $\lim _{n \rightarrow \infty} \nu^{n}=\nu$.

From the definition of $\nu$ and (85), we obtain that

$$
\nu-\nu^{n}-\frac{T}{n+1} \geq 0 .
$$

With (84), we have $T+M-\nu^{n}-\frac{T}{n+1} \geq 0$. Moreover, using (82) and (83), we obtain that $T+M-\nu^{n}>0$. Finally, $0 \leq \alpha^{n}<1$, which gives the result.

Proof of Lemma 4.7. Since the process $\left\{\eta_{t}^{n}\right\}$ is strictly increasing, it follows that $\gamma^{n}$ is the unique solution of the following equation:

$$
\eta_{\gamma^{n}}^{n}=T \text {. }
$$

However,

$$
\begin{aligned}
\eta_{\gamma}^{n} & =\eta_{\nu^{n}}^{n}+\gamma-\nu^{n}-\int_{\nu^{n}}^{\gamma}\left|\theta_{s}^{n}\right| d s \\
& =\frac{n T}{n+1}+\frac{T\left(\gamma-\nu^{n}\right)}{(n+1)\left(T+M-\nu^{n}\right)} .
\end{aligned}
$$

Using (15), it follows that

$$
\eta_{\gamma}^{n} \leq T
$$

From (86), we have

$$
\eta_{\gamma}^{n}+\gamma^{n}-\gamma=T
$$

Combining (87)-(89), we obtain (38).

By using the definition of $\left\{\theta_{t}^{n}\right\},(15)$, and the fact that $\left|\theta_{t}\right| \leq 1$, we have

$$
\begin{aligned}
\int_{0}^{\gamma}\left|\theta_{s}-\theta_{s}^{n}\right|^{2} d s & \leq 2\left[\int_{0}^{\nu^{n}}\left|\theta_{s}-\theta_{s}^{n}\right| d s+\int_{\nu^{n}}^{\nu}\left|\theta_{s}-\theta_{s}^{n}\right| d s+\int_{\nu}^{\gamma}\left|\theta_{s}-\theta_{s}^{n}\right| d s\right] \\
& \leq 2\left[\frac{T+M}{n+1}+\nu-\nu^{n}+\frac{T}{n+1} \frac{\gamma-\nu}{T+M-\nu^{n}}\right] .
\end{aligned}
$$


From (15), (82), and (83), it follows that

$$
\frac{\gamma-\nu}{T+M-\nu^{n}} \leq \frac{T+M-\nu}{T+M-\nu^{n}} \leq 1
$$

Consequently, there exists a constant $C_{1}$ such that

$$
E_{P}\left[\left|\int_{0}^{\gamma}\right| \theta_{s}-\left.\left.\theta_{s}^{n}\right|^{2} d s\right|^{2}\right] \leq C_{1}\left\{\frac{1}{(n+1)^{2}}+E_{P}\left[\left|\nu-\nu^{n}\right|^{2}\right]\right\} .
$$

Similarly, it is easy to deduce the same bound for $E_{P}\left[\int_{0}^{\gamma}\left|\eta_{s}-\eta_{s}^{n}\right|^{2} d s\right]$, which gives the result.

Acknowledgments. The authors are grateful to anonymous referees for many suggestions which have greatly improved the presentation of the paper.

The authors would like to thank P. Bertrand (L2S-CNRS) and F. Lamnhabi (NCN, L2S-CNRS) for their support.

\section{REFERENCES}

[1] A. Bressan And F. Rampazzo, Impulsive control systems with commutative vector fields, J. Optim. Theory Appl., 71 (1991), pp. 67-83.

[2] K. L. Chung, Lectures from Markov Processes to Brownian Motion, Springer-Verlag, New York, 1980.

[3] K. L. Chung And R. J. Williams, Introduction to Stochastic Integration, Birkhäuser Boston, Boston, 1990.

[4] F. Dufour AND B. Miller, On the correspondence between singular control and generalized control, in Proceedings of the 5th IFAC Symposium on Nonlinear Control Systems, St. Petersburg, Russia, 2001, pp. 1174-1178.

[5] N. El Karoui, H. Nguyen, and M. Jeanblanc-Picqué, Compactification methods in the control of degenerate diffusions: Existence of an optimal control, Stochastics Stochastics Rep., 20 (1987), pp. 169-219.

[6] R. J. Elliott, Stochastic Calculus and Applications, Springer-Verlag, New York, 1982.

[7] A. Friedman, Stochastic Differential Equations and Applications, Vol. 1, Academic Press, New York, 1975.

[8] U. G. Haussmann and J.-P. Lepeltier, On the existence of optimal controls, SIAM J. Control Optim., 28 (1990), pp. 851-902.

[9] U. G. Haussmann and W. Suo, Singular optimal stochastic controls I: Existence, SIAM J. Control Optim., 33 (1995), pp. 916-936.

[10] U. G. Haussmann AND W. SuO, Singular optimal stochastic controls II: Dynamic programming, SIAM J. Control Optim., 33 (1995), pp. 937-959.

[11] S. He, J. WAng, And J. YAn, Semimartingale Theory and Stochastic Calculus, Science Press, New York, 1992.

[12] I. Karatzas and S. E. Shreve, Brownian Motion and Stochastic Calculus, 2nd ed., SpringerVerlag, New York, 1991.

[13] N. V. Krylov, Controlled Diffusion Processes, Springer-Verlag, New York, 1980.

[14] P. A. Meyer, Probabilités et potentiel, Hermann, Paris, 1966.

[15] B. M. Miller, Method of discontinuous time change in problems of control for impulse and discrete-continuous systems, Automat. Remote Control, 54 (1993), pp. 1727-1750.

[16] B. M. MiLleR, The generalized solutions of nonlinear optimization problems with impulse control, SIAM J. Control Optim., 34 (1996), pp. 1420-1440.

[17] B. M. Miller And W. J. RungGaldier, Optimization of observations: A stochastic control approach, SIAM J. Control Optim., 35 (1997), pp. 1030-1052.

[18] Y. V. Orlov, Theory of Optimal Systems with Generalized Controls, Nauka, Moscow, 1988 (in Russian).

[19] P. ProtTer, Stochastic Integration and Differential Equations, Springer-Verlag, New York, 1990. 
[20] D. Revuz and M. Yor, Continuous Martingales and Brownian Motion, 3rd ed., SpringerVerlag, New York, 1999.

[21] J. Warga, Variational problems with unbounded controls, J. Soc. Indust. Appl. Math. Ser. A Control, 3 (1965), pp. 424-438.

[22] H. ZHU, Generalized solution in singular control: The nondegenerate problem, Appl. Math. Optim., 25 (1992), pp. 225-245. 